\title{
Pouvoir antioxydant et teneurs en composés phénoliques de deux espèces du genre Albertisia: Albertisia cordifolia (Mangenot \& J. Miège) Forman et Albertisia scandens (Mangenot \& J. Miège) Forman (Menispermaceae)
}

\author{
Diomande Awa, Doctorante \\ UFR Sciences de la Nature, \\ Université Nangui Abrogoua, Abidjan, Côte d'Ivoire \\ Yao Konan, Assistant
}

Centre Suisse de Recherche Scientifique en Côte d'Ivoire, Abidjan, Côte d'Ivoire/ Centre National de Floristique, Université Félix Houphouët-Boigny, Abidjan, Côte d'Ivoire/ Institut Botanique Aké-Assi d'Andokoi, Abidjan, Côte d'Ivoire

Sylla Youssouf, Doctorant

Tra Bi Fézan Honora, Professeur

UFR Sciences de la Nature,

Université Nangui Abrogoua, Abidjan, Côte d'Ivoire

Bakayoko Adama, Professeur

Kone Mamidou Witabouna, Professeur

UFR Sciences de la Nature, Université Nangui Abrogoua, Abidjan, Côte d'Ivoire/ Centre Suisse de Recherches Scientifiques en Côte d'Ivoire,

Abidjan, Côte d'Ivoire

Doi: 10.19044/esj.2018.v14n30p128 URL:http://dx.doi.org/10.19044/esj.2018.v14n30p128

\begin{abstract}
Albertisia cordifolia and Albertisia scandens are medicinal plants of Menispermaceae family, used in traditional medicine in Côte d'Ivoire. Both species were evaluated in vitro for antioxidant activity and contents of total polyphenols, flavonoids and condensed tannins. In the present study, six extracts (aqueous, ethyl acetate and ethanolic) were prepared from the leaves of $A$. cordifolia and the whole plant of $A$. scandens. The antioxidant assays were performed using the DPPH free radical scavenging and FRAP tests. Total polyphenols, flavonoids and condensed tannins were determined by spectrophotocolorimetric method. The results revealed that the yields of extraction are between 4.69-12.27\%. The percentages of DPPH inhibition
\end{abstract}


were between 33.84 and $69.21 \%$ while ascorbic acid and trolox gave $93.66 \%$ and $59.77 \%$ respectively. The $\mathrm{IC}_{50}$ values ranged from 20 to $280 \mu \mathrm{g} \mathrm{ml}^{-1}$ for DPPH. The ethyl acetate extract of $A$. cordifolia was the most active $\left(\mathrm{IC}_{50}=\right.$ $\left.20 \mu \mathrm{g} \mathrm{ml}^{-1}\right)$ compared to the other extracts and trolox $\left(28 \mu \mathrm{g} \mathrm{ml}^{-1}\right)$. The antioxidant capacities (DPPH) varied between 1.90 and $7.18 \mathrm{mg} \mathrm{ET} \mathrm{g}^{-1} \mathrm{MS}$. For FRAP, the extracts showed a dose-dependent activity with antioxidant

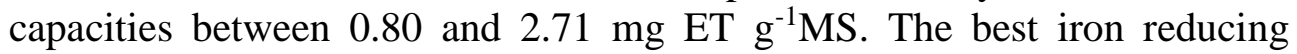
powers were obtained for the aqueous extract of $A$. scandens $(2.71 \pm 0.59 \mathrm{mg}$ $\mathrm{ET} \mathrm{g}^{-1} \mathrm{MS}$ ) and ethanolic extract of $A$. cordifolia. These results show that these two species may play a role in the prevention of oxidative stress related diseases.

Keywords: Albertisia cordifolia, Albertisia scandens, Menispermaceae, phenolic compounds, antioxidant activity

\section{Résumé}

Albertisia cordifolia et Albertisia scandens sont des plantes médicinales de la famille des Menispermaceae, utilisées en médecine traditionnelle en Côte d'Ivoire. Elles ont été évaluées, in vitro, pour leurs activités antioxydantes et leurs teneurs en polyphénols totaux, flavonoïdes et tanins condensés. Pour la présente étude, six extraits (aqueux, acétate d'éthyle et éthanoliques) ont été préparés, à partir des feuilles de $A$. cordifolia et de la plante entière de $A$. scandens. Le pouvoir antioxydant a été réalisé en utilisant les tests de piégeage du radical libre DPPH et FRAP. Les polyphénols totaux, les flavonoïdes et les tanins condensés ont été dosés par la méthode spectrophotocolorimétrique. Les résultats ont révélé que les rendements d'extraction sont compris entre $4,69 \%$ et $12,27 \%$. Les pourcentages d'inhibition du DPPH sont compris entre $33,84 \%$ et $69,21 \%$ tandis que l'acide ascorbique et le trolox ont donné, respectivement, $93,66 \%$ et $59,77 \%$. Les $\mathrm{CI}_{50}$ varient de 20 à $280 \mu \mathrm{g} . \mathrm{mL}^{-1}$ avec le DPPH. L'extrait acétate d'éthyle de A. cordifolia a été le plus actif $\left(\mathrm{CI}_{50}=20 \mu \mathrm{g} \cdot \mathrm{mL}^{-1}\right)$ que le trolox $\left(28 \mu \mathrm{g} \cdot \mathrm{mL}^{-}\right.$ $\left.{ }^{1}\right)$. Les capacités antioxydantes (DPPH) sont comprises entre 1,90 et 7,18 mgET.g ${ }^{-1}$ MS. Pour le FRAP, les extraits ont montré une activité dosedépendante avec des capacités comprises entre 0,80 et $2,71 \mathrm{mgET} . \mathrm{g}^{-1} \mathrm{MS}$. Les meilleurs pouvoirs réducteurs du fer ont été obtenus avec les extraits aqueux de $A$. scandens $\left(2,71 \pm 0,59 \mathrm{mgET} . \mathrm{g}^{-1} \mathrm{MS}\right)$ et éthanolique de $A$. cordifolia. Ces résultats montrent que ces deux espèces peuvent jouer un rôle dans la prévention des maladies liées aux stress oxydatif.

Mots-clés: Albertisia cordifolia, Albertisia scandens, Menispermaceae, composés phénoliques, activité antioxydante 


\section{Introduction}

Albertisia cordifolia et Albertisia scandens sont deux espèces végétales rares et endémiques respectivement du Sud-Est de la région côtière de Côte d'Ivoire et de l'Afrique tropical de l'Ouest. Ces deux espèces de la famille des Menispermaceae sont utilisées en médecine traditionnelle en Côte d'Ivoire pour traiter plusieurs maladies (infection cutanée, sous-cutanée, anémie, les œdèmes aux jambes, etc..). La plupart des maladies sont causées par le stress oxydant, qui suscite la recherche de nouveaux remèdes antioxydants. Le stress oxydatif provoque des dommages importants qui sont des accélérations du vieillissement cellulaire. Ce vieillissement aboutit à des pathologies graves telles que les maladies cardiovasculaires et neurodégénératives, le cancer, le diabète, le syndrome métabolique et les maladies digestives (Aseervatham et al., 2013). Plusieurs situations de la vie quotidienne entraînent la production de radicaux libres responsables du stress oxydatif. Il s'agit, par exemple, de la surproduction endogène d'agents prooxydants d'origine inflammatoire, la carence en un ou plusieurs antioxydants apportés par la nutrition comme les vitamines ou les oligoéléments (Sohal et al., 2002; Favier, 2003) ou même une exposition environnementale à des facteurs prooxydants tels que le tabac, 1'alcool, les médicaments et les pesticides (Sohal et al., 2002 ; Magder, 2006). Pour faire face à ce désordre, les antioxydants sont préconisés, les plus utilisés étant ceux de synthèse. Cependant, l'utilisation des molécules antioxydantes synthétiques disponibles est, actuellement, remise en cause en raison des risques potentiels pour la santé et de la toxicité qu'elles sont capables de provoquer (Kicel et al., 2016 ; Liu \& Yang, 2018). C'est pourquoi, l'accent est, de plus en plus, mis sur la recherche de nouvelles sources d'antioxydants dont les plantes médicinales (Liu et al., 2017 ; Wang et al., 2018). De nombreuses études ont montré que ces dernières possèdent des propriétés antioxydantes (Bokhari et al., 2013 ; Konan et al., 2014 ; Afsar et al., 2018). De plus, plusieurs espèces végétales de la famille des Menispermaceae sont douées d'activité antioxydante très importante. C'est le cas, par exemple, de Cyclea peltata (Meena \& Santhy, 2015) et Cyclea gracillima (Lin et al., 2018). La présente étude a, ainsi, retenu dans la flore ivoirienne, deux espèces du genre Albertisia appartenant à cette famille. Il s'agit de Albertisia cordifolia et Albertisia scandens. Le macéré des racines de $A$. cordifolia est utilisé en boisson ou en lavement pour ses propriétés stimulantes et aphrodisiaques ainsi que pour ses activités calmante et décongestive. Quant à Albertisia scandens, le suc de ses feuilles est utilisé contre certaines dermatoses (Bouquet \& Debray, 1974). Les travaux rares, sur les activités antioxydantes de ces deux plantes et la possibilité que les propriétés thérapeutiques mentionnées reposent sur la capacité de piégeage des radicaux libres des différents extraits justifient le choix de celles-ci. 
Cette étude vise à évaluer in vitro l'activité antioxydante des extraits aqueux, acétate d'éthyle et éthanoliques de ces deux espèces de la famille des Menispermaceae, à l'aide de la méthode de piégeage du radical libre DPPH et celle de la réduction du fer FRAP. Les résultats pourraient permettre une valorisation de ces espèces en médecine traditionnelle en Côte d'Ivoire.

\section{Matériel et méthodes}

\section{Récolte des organes étudiés}

Les feuilles de Albertisia cordifolia et la plante entière de Albertisia scandens ont constituées l'essentiel du matériel végétal utilisé au cours de cette étude. Elles ont été récoltées dans la forêt de Anguédédou, dans la commune de Yopougon (Sud Côte d' Ivoire) en Mai 2016. Elles ont, ensuite, été séchées, au laboratoire, sous climatisation à $18^{\circ} \mathrm{C}$ pendant deux semaines puis rendus en poudre.

\section{Extraction}

A $10 \mathrm{~g}$ de poudre végétale, ont été ajoutés $100 \mathrm{~mL}$ d'eau distillée. L'ensemble a été porté à ébullition pendant $15 \mathrm{~min}$. Les extraits ont été filtrés sur du coton hydrophile et du papier filtre. Les filtrats obtenus ont été séchés à sec dans une étuve ventilée à $37^{\circ} \mathrm{C}$.

D'autres extraits ont été préparés à partir d'une extraction successive par des solvants de polarité croissante à raison de $10 \mathrm{~g}$ de poudre végétale dans $100 \mathrm{~mL}$ d'acétate d'éthyle pendant 24 heures sous agitateur mécanique. Après filtration, on a obtenu l'extrait acétate d'éthyle. Le marc séché a été repris dans $100 \mathrm{~mL}$ d'éthanol (90\%). Les filtrats acétate d'éthyle et éthanolique ont également été séchés dans une étuve ventilée à $37^{\circ} \mathrm{C}$.

\section{Calcul du rendement}

Le rendement d'extraction a été obtenu par la formule suivante :

$$
\mathbf{R}(\%)=(\mathbf{M e} / \mathbf{M v}) \times \mathbf{1 0 0}
$$

Avec R $(\%)=$ Rendement de l'extraction en $\%, \mathrm{Me}=$ Masse de l'extrait après l'évaporation du solvant, $\mathrm{Mv}=$ Masse de la matière végétale utilisée pour l'extraction.

\section{Evaluation de l'activité antioxydante}

Deux méthodes ont été utilisées pour l'évaluation de l'activité antioxydante des extraits ; le test du DPPH (1,1-diphényl-2-picrylhydrazyl) et le test FRAP (ferric reducing antioxydant power). 


\section{Préparation des solutions d'extraits végétaux à tester}

Des solutions mères méthanoliques à $26 \mathrm{mg} \cdot \mathrm{mL}^{-1}$ ont été préparées. Pour les tests, une gamme de concentrations ( 500 à $\left.31,25 \mu \mathrm{g} . \mathrm{mL}^{-1}\right)$ pour le test DPPH et $\left(31,25\right.$ à $\left.0,976 \mu \mathrm{g} \cdot \mathrm{mL}^{-1}\right)$ pour le test FRAP a été préparée dans le méthanol.

\section{Test de piégeage du radical 2,2-diphényl-1-picrylhydrazyle (DPPH)}

Les tests ont été réalisés selon le protocole décrit par Kosanić \& Ranković (2011). Cent microlitres $(100 \mu \mathrm{L})$ des différentes dilutions (500 à $31,25 \mu \mathrm{g} \cdot \mathrm{mL}^{-1}$ ) ont été ajoutés à $2500 \mu \mathrm{L}$ de solution méthanolique de DPPH $(0,4 \%)$. Après incubation pendant $30 \mathrm{~min}$ à $30^{\circ} \mathrm{C}$, à l'obscurité, l'absorbance a été mesurée à $517 \mathrm{~nm}$ en utilisant la solution méthanolique de DPPH comme blanc $(100 \mu \mathrm{L}$ de méthanol dans $2500 \mu \mathrm{L}$ de solution DPPH). Deux essais ont été réalisés pour chaque concentration, puis la moyenne des deux mesures a été calculée. L'acide ascorbique et le trolox pris comme contrôles positifs ont été préparés dans les mêmes conditions que les échantillons. Une courbe d'étalonnage a été tracée avec des concentrations de trolox allant de 800 à 50 $\mu \mathrm{g} . \mathrm{mL}^{-1}$. Le pourcentage d'inhibition du radical DPPH a été obtenu par la formule suivante :

\section{\% Inhibition $(\mathrm{DPPH})=[(\mathbf{A 0}-\mathbf{A 1}) / \mathrm{A1}] \times \mathbf{1 0 0}$}

Avec $\mathrm{A} 0=$ Absorbance du blanc, $\mathrm{A} 1=$ Absorbance de l'extrait à une concentration donnée.

La concentration inhibitrice $50 \%\left(\mathrm{CI}_{50}\right)$ est la concentration des antioxydants nécessaire pour réduire $50 \%$ du radical DPPH. Elle a été déterminée graphiquement par la régression linéaire. Selon Sahgal et al. (2009), une faible valeur de $\mathrm{CI}_{50}$ d'un extrait indique sa forte activité antioxydante. Les capacités antioxydantes $\left(\mathrm{mgET} . \mathrm{g}^{-1} \mathrm{MS}\right)$ des extraits ont été déterminées par la formule suivant (Wangcharoen \& Morasuk, 2007) :

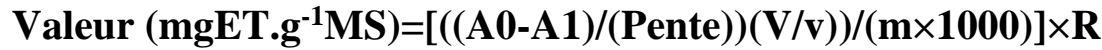

Avec $\mathrm{Ae}=$ Valeur de l'absorbance de l'échantillon, $\mathrm{A} 0=$ Valeur de l'absorbance du témoin, Pente $=$ Pente de la droite de régression exprimant les valeurs des absorbances en fonction des différentes concentrations de trolox, $\mathrm{V}=$ Volume total d'extrait préparé pour obtenir la solution mère, $\mathrm{v}=$ Volume de l'extrait prélevé pour le test, $\mathrm{m}=$ Masse de l'échantillon prélevé pour préparer la solution mère, $\mathrm{R}=$ Rendement de l'extrait, 1000, Facteur de conversion ( $\mu \mathrm{g}$ en $\mathrm{mg})$. 


\section{Test de réduction du fer (FRAP)}

Le pouvoir réducteur du fer $\left(\mathrm{Fe}^{3+}\right)$ dans les extraits est déterminé selon la méthode décrite par Oyaizu (1986). Le réactif FRAP est obtenu en mélangeant une solution de TPTZ $(10 \mathrm{nM})$, une solution tampon d'acétate de sodium $(\mathrm{pH} 3,6)$ et une solution $\mathrm{FeCl}_{3}(20 \mathrm{nM})$ dans les proportions 1:10:1. Une quantité de $100 \mu \mathrm{L}$ de chaque extrait à différentes concentrations $(31,25$ à $0,976 \mu \mathrm{g} \cdot \mathrm{mL}^{-1}$ ) est mélangé avec $2500 \mu \mathrm{L}$ de réactif FRAP et incubé à l'obscurité pendant $30 \mathrm{~min}$. L'absorbance du produit coloré (complexe tripyridyltriazine ferreux) a été mesurée à $593 \mathrm{~nm}$. Le trolox pris comme contrôle positif a été préparé dans les mêmes conditions que les échantillons. Une courbe d'étalonnage a été établie avec le trolox dont les concentrations varient de 800 à $50 \mu \mathrm{g} \cdot \mathrm{mL}^{-1}$. La capacité antioxydante des différents extraits est exprimée en mg de trolox équivalent/g de matière sèche (mg de ET. $\mathrm{g}^{-1} \mathrm{MS}$ ) suivant la formule précédente.

\section{Détermination de la teneur en composés phénoliques}

Polyphénols totaux

La teneur en polyphénols totaux des extraits a été déterminée par la méthode de Folin-Ciocalteu (Singleton \& Rossi, 1965). Un volume de 2500 $\mu \mathrm{L}$ d'une solution de Folin-Ciocalteu (dilué au 1/10 ème) est ajouté à $500 \mu \mathrm{L}$ d'extrait et l'ensemble est incubé pendant 2 min à température ambiante. Puis, $2000 \mu \mathrm{L}$ de bicarbonate de sodium à 75 g. $\mathrm{L}^{-1}$ y sont ajoutés, suivi immédiatement d'une incubation à $50{ }^{\circ} \mathrm{C}$ pendant $15 \mathrm{~min}$. Après le refroidissement rapide des tubes dans de l'eau glacée, la lecture est effectuée contre un blanc à l'aide d'un spectrophotomètre à $760 \mathrm{~nm}$. La courbe d'étalonnage a été réalisée avec l'acide gallique à des concentrations comprises entre 50 à $0 \mu \mathrm{g} \cdot \mathrm{mL}^{-1}$. Les résultats sont exprimés en $\mu \mathrm{g}$ équivalent d'acide gallique (AG) par gramme de matière sèche $\left(\mu \mathrm{gEAG} \cdot \mathrm{g}^{-1} \mathrm{MS}\right)$ en utilisant la formule suivante (Wangcharoen \& Morasuk, 2007) :

\section{Teneur $\left(\mu \mathrm{gES} . \mathrm{g}^{-1} \mathrm{MS}\right)=[((\mathrm{A1}-\mathrm{A0}) /($ Pente $)(\mathrm{V} / \mathrm{v})) / \mathrm{m}] \times \mathbf{R}$}

Avec ES : Equivalent de standard; A1 = Valeur de l'absorbance de l'échantillon ; A0 = Valeur de l'absorbance du témoin ; Pente $=$ Pente de la droite de régression exprimant les valeurs des absorbances en fonction des différentes concentrations du standard; $\mathrm{V}=$ Volume total d'extrait préparé pour obtenir la solution mère; $v=$ Volume de l'extrait prélevé pour le test ; $\mathrm{m}$ $=$ Masse de l'échantillon prélevé pour préparer la solution mère ; $\mathrm{R}=$ Rendement de l'extrait.

Flavonoïdes

La teneur en flavonoïdes des extraits a été déterminée en utilisant la méthode colorimétrique au trichlorure d'aluminium (Bahorun et al., 1996). A 
$1 \mathrm{~mL}$ de chaque extrait à $500 \mu \mathrm{g} \cdot \mathrm{mL}^{-1}$ a été ajouté $1 \mathrm{~mL}$ d'une solution d' $\mathrm{AlCl}_{3}$ ( $2 \%$ dans le méthanol). Au moyen d'un vortex, le mélange obtenu est vigoureusement agité et l'absorbance est lue à $430 \mathrm{~nm}$ après $10 \mathrm{~min}$ d'incubation. Les tests ont été effectués trois fois. L’équation de la courbe d'étalonnage $(Y=0,0318 \mathrm{X}+0,0193)$ a été utilisée. Les résultats sont exprimés en microgrammes d'équivalent de quercétine (EQ) par milligramme d'extrait ( $\left.\mu \mathrm{gEQ} \cdot \mathrm{g}^{-1} \mathrm{MS}\right)$ en utilisant la formule ci-dessus.

\section{Tanins condensés}

Le dosage des tanins condensés est réalisé selon la méthode de la vanilline en milieu acide (Ba et al., 2010). Le réactif de la vanilline a été préparé en mélangeant, à volume égal, du $\mathrm{HCl}$ à $8 \%(\mathrm{v} / \mathrm{v})$, du méthanol à $37 \%$ $(\mathrm{v} / \mathrm{v})$ et $4 \%$ de vanilline dans du méthanol $(\mathrm{m} / \mathrm{v})$. Le mélange a été maintenu à $30{ }^{\circ} \mathrm{C}$ avant le dosage. Un volume de $200 \mu \mathrm{L}$ de chaque extrait à analyser a été ajouté à $1000 \mu \mathrm{L}$ de réactif de vanilline ; le mélange a été agité puis incubé à l'obscurité à $30^{\circ} \mathrm{C}$ pendant $20 \mathrm{~min}$. L'absorbance est mesurée à $500 \mathrm{~nm}$ au spectrophotomètre UV (Perkin Elmer) contre un blanc constitué d'un mélange de méthanol $(37 \%)$ et de $\mathrm{HCl}(8 \%)$ à volume égal.

La courbe d'étalonnage de la catéchine a été construite à partir des concentrations comprises entre 300 et $0 \mu \mathrm{g} \cdot \mathrm{mL}^{-1}$. Les résultats ont été exprimés en $\mu \mathrm{g}$ équivalent de catéchine (EC) par gramme de matière sèche ( $\left.\mu \mathrm{gEC} . \mathrm{g}^{-1} \mathrm{MS}\right)$ en utilisant la formule précédente.

\section{Analyses statistiques}

L'analyse de variance à un critère de classification (ANOVA 1) a été utilisée pour comparer le pourcentage d'inhibition du DPPH des différents extraits, 1'activité de FRAP et les teneurs en polyphénols totaux, flavonoïdes et tanins condensés à l'aide du logiciel STATISTICA 7.1. Lorsque la comparaison a montré une différence significative entre les extraits testés, le test de comparaison multiple des moyennes est effectué (test de Turkey) pour déterminer le niveau de différence entre les différents groupes d'extraits (Westlake, 1971). Si $\mathrm{P}<0,05$ le test était significatif. Les corrélations $\left(\mathrm{R}^{2}\right)$ entre les concentrations et les pourcentages d'inhibition de DPPH des extraits ont été déterminées à partir des courbes dose-réponse, $\mathrm{R}^{2} \geq 0,90$ est considéré comme fortement corrélé (Kaur et al., 2008). Les résultats ont été exprimés en moyennes \pm déviation standard.

\section{Résultats}

\section{Rendements d'extraction}

La figure 1 illustre les résultats des rendements d'extraction qui varient de 4,69\% à 12,27\%. L'extrait brut aqueux des plantes entières de A. scandens a enregistré un fort rendement de l'ordre de $12,27 \%$ suivi de l'extrait 
éthanolique avec 9,12\%. L'extrait brut aqueux des feuilles de $A$. cordifolia a donné le plus faible rendement, de 4,69\%. Pour cette espèce, les meilleurs rendements d'extraction $(7,57 \%)$ ont été obtenus avec l'éthanol suivi de l'acétate d'éthyle $(5,16 \%)$.
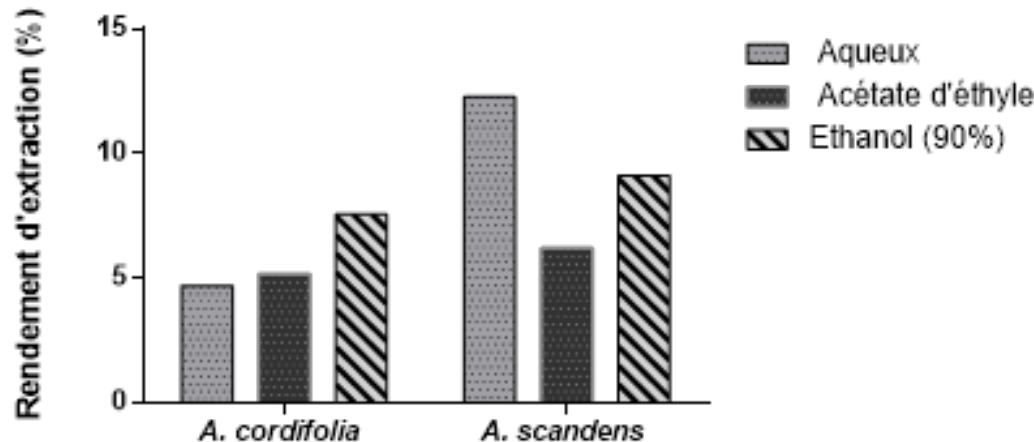

Figure 1 : Rendements des différents types d'extraits de feuilles de A. cordifolia et de plantes entières de $A$. scandens.

\section{Activité antioxydante}

Tous les extraits ont montré des activités antioxydantes avec les deux méthodes. Les résultats obtenus sont présentés dans les tableaux 1 et 2.

\section{Activité antiradicalaire au DPPH}

Sur les six extraits bruts testés, trois extraits $(50 \%)$ ont présenté des pourcentages d'inhibition supérieurs à $50 \%$. Une différence significative $(\mathrm{F}=$ $6,21 ; \mathrm{P}<0,001)$ a été observée entre les pourcentages d'inhibition du radical DPPH. Les pourcentages d'inhibition des différents extraits de $A$. cordifolia et A. scandens et des standards sont illustrés par la figure 2. Des pourcentages d'inhibition de 69,21 7,97\% pour l'extrait acétate d'éthyle de $A$. cordifolia et $53,29 \pm 11,47 \%$ pour l'extrait aqueux de $A$. scandens ont été obtenus. Un faible pourcentage d'inhibition a été enregistré avec les extraits éthanolique $(33,84 \pm$ $9,62 \%)$ et acétate d'éthyle $(37,79 \pm 5,57 \%)$ de $A$. scandens suivi de l'extrait aqueux des feuilles de $A$. cordifolia $(48,55 \pm 8,34 \%)$. Les capacités antioxydantes les plus élevées ont été obtenues avec les extraits aqueux de $A$. scandens $\left(8,33 \pm 1,79 \mathrm{mgET} . \mathrm{g}^{-1} \mathrm{MS}\right)$ suivi des extraits acétate d'éthyle $\left(7,18 \pm 0,83 \mathrm{mgET} \cdot \mathrm{g}^{-1} \mathrm{MS}\right)$ et éthanolique $\left(7,17 \pm 0,96 \mathrm{mgTE} \cdot \mathrm{g}^{-1} \mathrm{MS}\right)$ de $A$. cordifolia. Les capacités antioxydantes (Tableau 1) de ces trois extraits ne sont pas statistiquement différentes. Ces extraits ont donc des pouvoirs antioxydants superposables. Pour les extraits ayant enregistrés de faibles

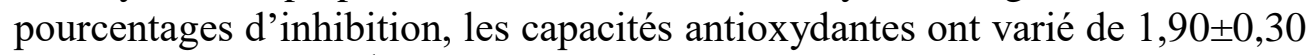
à 4,80 $\pm 1,36 \mathrm{mgET} \cdot \mathrm{g}^{-1} \mathrm{MS}$.

Tous les extraits actifs ont donné des $\mathrm{CI}_{50}$ supérieures à celle de l'acide ascorbique $\left(\mathrm{CI}_{50}=17 \mu \mathrm{g} \cdot \mathrm{mL}^{-1}\right)$. Seul l'extrait acétate d'éthyle des 
feuilles de $A$. cordifolia $\left(\mathrm{CI}_{50}=20 \mu \mathrm{g} \cdot \mathrm{mL}^{-1}\right)$ a enregistré une $\mathrm{CI}_{50}$ inférieure à celle du trolox $\left(28 \mu \mathrm{g} \cdot \mathrm{mL}^{-1}\right)$.

Une forte corrélation logarithmique positive a été obtenue entre les concentrations des extraits et les pourcentages d'inhibition pour l'extrait éthanolique de $A$. scandens $\left(\mathrm{R}^{2}=0,9173\right)$, c'est à dire que l'activité de cet extrait augmente au fur et à mesure que la concentration augmente. Il n'y a pas de corrélation entre les différentes concentrations et les activités antiradicalaires pour les cinq autres extraits végétaux testés. Ces derniers suivent un comportement polynomial de degré divers. Autrement dit, pour un extrait, l'intensité de l'activité est indépendante de la concentration.

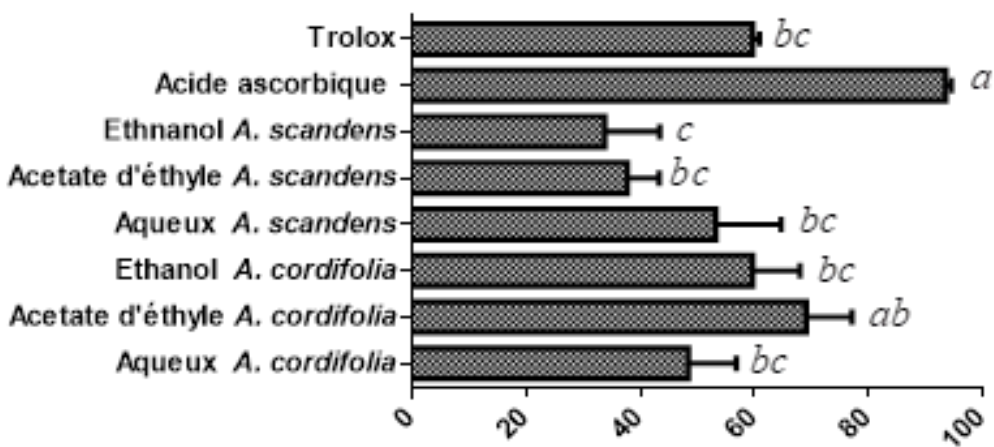

Figure 2 : Pourcentage d'inhibition des extraits de A. cordifolia, A. scandens et des standards.

Tableau 1 : Activité de piégeage des radicaux libres DPPH des extraits de A. cordifolia et A. scandens et des standards.

\begin{tabular}{|c|c|c|c|c|c|}
\hline \multirow[b]{2}{*}{$\begin{array}{l}\text { Espèces } \\
\text { végétales et } \\
\text { composés de } \\
\text { références }\end{array}$} & \multirow[b]{2}{*}{$\begin{array}{l}\text { Organe } \\
\text { utilisé }\end{array}$} & \multirow[b]{2}{*}{ Extrait } & \multicolumn{2}{|c|}{ Potentiel antioxydant } & \multirow[b]{2}{*}{$\overline{\mathbf{R}^{2}}$} \\
\hline & & & $\begin{array}{l}\text { Capacité } \\
\text { antiradicalaire } \\
\text { DPPH (mgTE.g } \\
{ }^{1} \text { MS } \pm \text { SD) }\end{array}$ & $\begin{array}{l}\text { CI } I_{50} \\
\left(\mu \mathrm{g} . \mathrm{mL}^{-1}\right)\end{array}$ & \\
\hline $\begin{array}{l}\text { Albertisia } \\
\text { cordifolia }\end{array}$ & Feuille & $\begin{array}{l}\text { Aqueux } \\
\text { Acétate d'éthyle } \\
\text { Ethanol }\end{array}$ & $\begin{array}{l}2,16 \pm 0,37^{\mathrm{b}} \\
7,18 \pm 0,83^{\mathrm{a}} \\
7,17 \pm 0,96^{\mathrm{a}}\end{array}$ & $\begin{array}{l}123,5 \\
20 \\
88\end{array}$ & $\begin{array}{l}0,998 \\
0,986 \\
0,979\end{array}$ \\
\hline $\begin{array}{l}\text { Albertisia } \\
\text { scandens }\end{array}$ & $\begin{array}{l}\text { Plante } \\
\text { entière }\end{array}$ & $\begin{array}{l}\text { Aqueux } \\
\text { Acétate d'éthyle } \\
\text { Ethanol }\end{array}$ & $\begin{array}{l}8,33 \pm 1,79^{\mathrm{a}} \\
1,90 \pm 0,30^{\mathrm{b}} \\
4,80 \pm 1,36^{\mathrm{ab}}\end{array}$ & $\begin{array}{l}105 \\
162 \\
279\end{array}$ & $\begin{array}{l}0,917 \\
0,901 \\
0,993\end{array}$ \\
\hline $\begin{array}{l}\text { Acide } \\
\text { ascorbique } \\
\text { Trolox }\end{array}$ & & & $\begin{array}{l}\text { nd } \\
\text { nd }\end{array}$ & $\begin{array}{l}17 \\
28\end{array}$ & $\begin{array}{l}0,9983 \\
0,8510\end{array}$ \\
\hline $\begin{array}{l}\text { Paramètres } \\
\text { statistiques }\end{array}$ & & $\begin{array}{l}\text { ddl } \\
\text { F } \\
P\end{array}$ & $\begin{array}{l}5 \\
6,58 \\
<0,001\end{array}$ & & \\
\hline
\end{tabular}

Les barres portant la même lettre sont statistiquement identiques $(\alpha=5 \%)$

TE : trolox équivalent, $\mathrm{R}$ : coefficient de corrélation, SD : déviation standard, ddl : degré de liberté, F : statistique de Fisher, $\mathrm{P}$ : probabilité, nd : non déterminé. Les valeurs avec les mêmes lettres dans chaque colonne ne sont pas significativement différentes $(\mathrm{p}<0,05)$. 


\section{Pouvoir réducteur du fer (FRAP)}

Les graphes de la figure 3 présentent le pouvoir réducteur des extraits de feuilles de A. cordifolia (A) et A. scandens (B). Tous les extraits ont une activité dose dépendante. Cependant, tous les extraits présentent des pouvoirs réducteurs inférieurs à celui du produit de référence (trolox).

A

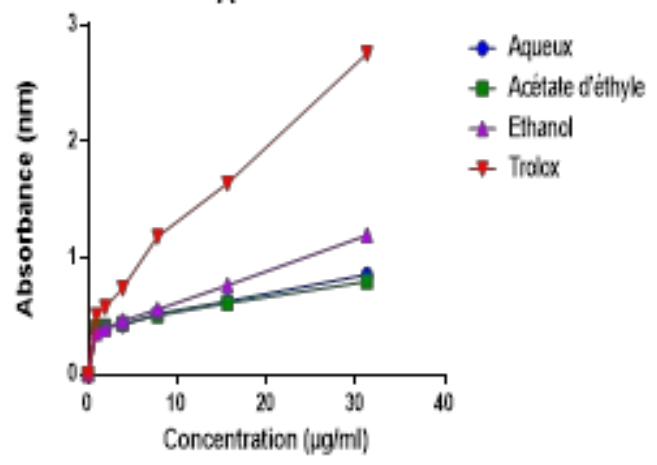

B

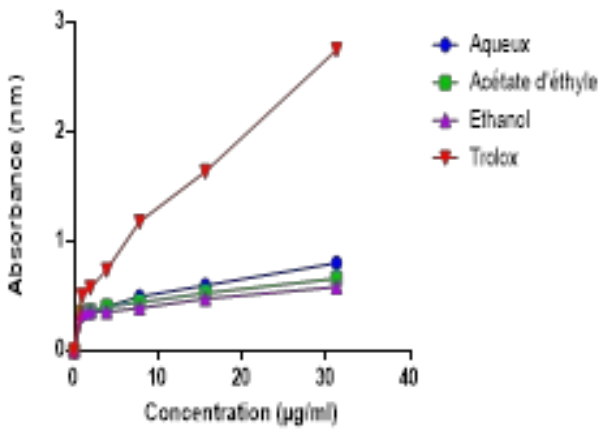

Figure 3 : Pouvoir réducteur du fer des extraits de A. cordifolia (A) et A. scandens $\mathbf{( B )}$.

Les activités réductrices du fer (Tableau 2) des deux plantes se situent entre 0,80 et $2,71 \mathrm{mgET} \cdot \mathrm{g}^{-1} \mathrm{MS}$. Une différence significative $(\mathrm{F}=7,04 ; \mathrm{P}$ $<0,001)$ a été observée entre la capacité de réduction du fer des extraits. L'extrait aqueux des plantes entières de $A$. scandens $\left(2,71 \pm 0,59 \mathrm{mgET} . \mathrm{g}^{-1} \mathrm{MS}\right)$ a enregistré la capacité de réduction du fer la plus élevée suivi des extraits éthanoliques $\left(2,49 \pm 0,58 \mathrm{mgET} . \mathrm{g}^{-1} \mathrm{MS}\right)$ et acétate d'éthyle $\left(1,34 \pm 0,19 \mathrm{mgET} . \mathrm{g}^{-}\right.$ ${ }^{1} \mathrm{MS}$ ) des feuilles de $A$. cordifolia.

Tableau 2 : Capacité de réduction du fer de A. cordifolia et A. scandens.55

\begin{tabular}{|c|c|c|c|}
\hline \multirow[b]{2}{*}{$\begin{array}{l}\text { Espèces végétales et } \\
\text { composés de référence }\end{array}$} & \multirow[b]{2}{*}{$\begin{array}{l}\text { Organe } \\
\text { utilisé }\end{array}$} & \multirow[b]{2}{*}{ Extrait } & \multirow{2}{*}{$\begin{array}{l}\text { Potentiel antioxydant } \\
\text { Capacité de réduction du } \\
\text { fer }\left(\mathrm{mgET} . \mathrm{g}^{-1} \mathrm{MS} \pm \mathrm{SD}\right)\end{array}$} \\
\hline & & & \\
\hline \multirow[t]{3}{*}{ Albertisia cordifolia } & Feuille & Aqueux & $1,09 \pm 0,24^{\mathrm{bc}}$ \\
\hline & & Acétate d'éthyle & $1,34 \pm 0,19^{a b c}$ \\
\hline & & Ethanol & $2,49 \pm 0,58^{\mathrm{ab}}$ \\
\hline \multirow[t]{3}{*}{ Albertisia scandens } & Plante & Aqueux & $2,71 \pm 0,59^{\mathrm{a}}$ \\
\hline & entière & Acétate d'éthyle & $0,80 \pm 0,18^{c}$ \\
\hline & & Ethanol & $0,92 \pm 0,21^{\mathrm{c}}$ \\
\hline \multirow{3}{*}{$\begin{array}{l}\text { Paramètres } \\
\text { statistiques }\end{array}$} & & ddl & 5 \\
\hline & & $\mathbf{F}$ & 7,04 \\
\hline & & $\mathbf{P}$ & $<0,001$ \\
\hline
\end{tabular}

SD : déviation standard, ddl : degré de liberté, F : statistique de Fisher, $\mathrm{P}:$ probabilité. Les valeurs avec les mêmes lettres dans chaque colonne ne sont pas significativement différentes $(\mathrm{p}<0,05)$. 


\section{Teneurs en composés phénoliques}

Le tableau 3 donne les teneurs en polyphénols totaux, flavonoïdes et tanins condensés. Les teneurs en polyphénols totaux sont comprises entre $10,64 \pm 0,16$ et $32,67 \pm 0,45 \mu \mathrm{gEAG} \cdot \mathrm{g}^{-1} \mathrm{MS}$. Une différence significative $(\mathrm{F}=$ $97,11 ; \mathrm{P}<0,001)$ a été observée entre les extraits. Les extraits acétate d'éthyle des feuilles de $A$. cordifolia $\left(32,67 \pm 0,45 \mu \mathrm{gEAG} . \mathrm{g}^{-1} \mathrm{MS}\right)$ et aqueux de $A$. scandens $\left(24,51 \pm 1,40 \mu \mathrm{gEAG} . \mathrm{g}^{-1} \mathrm{MS}\right)$ ont été les plus riches en polyphénols suivis des extraits éthanoliques $\left(21,86 \pm 0,04 \mu \mathrm{gEAG} . \mathrm{g}^{-1} \mathrm{MS}\right)$ de $A$. scandens et des feuilles de A. cordifolia $\left(21,56 \pm 1,10 \mu \mathrm{gEAG} . \mathrm{g}^{-1} \mathrm{MS}\right)$.

Les teneurs en flavonoïdes des extraits ont varié de 2,99 à 19,87 $\mu \mathrm{gEQ} \cdot \mathrm{g}^{-1} \mathrm{MS}$. Du point de vu statistique, une différence significative ( $\mathrm{F}=$ $89,48 ; \mathrm{P}<0,001)$ a été observée entre les extraits. Les extraits éthanoliques des feuilles de $A$. cordifolia $\left(19,87 \pm 1,68 \mu \mathrm{gEQ} . \mathrm{g}^{-1} \mathrm{MS}\right)$ et acétate d'éthyle des plantes entière de $A$. scandens $\left(17,65 \pm 0,50 \mu \mathrm{gEQ} \cdot \mathrm{g}^{-1} \mathrm{MS}\right)$ ont été plus riches en flavonoïdes suivis de l'extrait acétate d'éthyle des feuilles de A. cordifolia $\left(10,25 \pm 0,13 \mu \mathrm{gEQ} \cdot \mathrm{g}^{-1} \mathrm{MS}\right)$.

Les tanins condensés sont présents dans les extraits avec une différence significative entre les teneurs $(\mathrm{F}=1619,13 ; \mathrm{P}<0,001)$.

Les teneurs en tanins condensés des extraits ont varié de 4,69 à 725,41 $\mu \mathrm{gEC} . \mathrm{g}-$ ${ }^{1}$ MS. L'extrait acétate d'éthyle des feuilles de A. cordifolia $(725,41 \pm 11,97$ $\mu \mathrm{gEC} . \mathrm{g}^{-1} \mathrm{MS}$ ) s'est révélé le plus riche en tanins condensés suivi des extraits acétate d'éthyle $\left(500,13 \pm 9,40 \mu \mathrm{gEC} \cdot \mathrm{g}^{-1} \mathrm{MS}\right)$ et éthanolique $\left(113,50 \pm 9,38 \mu \mathrm{gEC} \cdot \mathrm{g}^{-1} \mathrm{MS}\right)$ des plantes entières de A. scandens.

Tableau 3 : Teneurs en polyphénols totaux, flavonoïdes et tanins condensés des extraits de A. cordifolia et $A$. scandens.

\begin{tabular}{|c|c|c|c|c|c|}
\hline \multirow[b]{2}{*}{$\begin{array}{l}\text { Espèces } \\
\text { végétales }\end{array}$} & \multirow[b]{2}{*}{$\begin{array}{l}\text { Organe } \\
\text { utilisé }\end{array}$} & \multirow[b]{2}{*}{ Extraits } & \multicolumn{3}{|c|}{ Teneurs } \\
\hline & & & 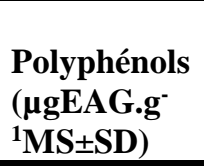 & $\begin{array}{l}\text { Flavonoïdes } \\
\text { ( } \mu \text { gEQ.g- } \\
{ }^{1} \mathbf{M S} \pm \text { SD) }\end{array}$ & $\begin{array}{l}\text { Tanins } \\
\text { condensés } \\
\left(\mu g E C . g^{-}\right. \\
\left.{ }^{1} \mathrm{MS} \pm \mathrm{SD}\right) \\
\end{array}$ \\
\hline $\begin{array}{l}\text { Albertisia } \\
\text { cordifolia }\end{array}$ & Feuille & $\begin{array}{l}\text { Aqueux } \\
\text { Acétate } \\
\text { d'éthyle } \\
\text { Ethanol }\end{array}$ & $\begin{array}{l}10,64 \pm 0,16^{\mathrm{d}} \\
32,67 \pm 0,45^{\mathrm{a}} \\
21,56 \pm 1,10^{\mathrm{b}}\end{array}$ & $\begin{array}{l}9,32 \pm 0,23^{b} \\
10,25 \pm 0,13^{b} \\
19,87 \pm 1,68^{a}\end{array}$ & $\begin{array}{l}4,69 \pm 0,68^{\mathrm{d}} \\
725,41 \pm 11,97^{\mathrm{a}} \\
99,04 \pm 1,26^{\mathrm{c}}\end{array}$ \\
\hline $\begin{array}{l}\text { Albertisia } \\
\text { scandens }\end{array}$ & $\begin{array}{l}\text { Plante } \\
\text { entière }\end{array}$ & $\begin{array}{l}\text { Aqueux } \\
\text { Acétate } \\
\text { d'éthyle } \\
\text { Ethanol }\end{array}$ & $\begin{array}{l}24,51 \pm 1,40^{\mathrm{b}} \\
16,65 \pm 0,02^{\mathrm{c}} \\
21,86 \pm 0,04^{\mathrm{b}}\end{array}$ & $\begin{array}{l}3,84 \pm 0,13^{\mathrm{c}} \\
17,65 \pm 0,50^{\mathrm{a}} \\
2,99 \pm 0,23^{\mathrm{c}}\end{array}$ & $\begin{array}{l}17,38 \pm 2,71^{\mathrm{d}} \\
500,13 \pm 9,40^{\mathrm{b}} \\
113,50 \pm 9,38 \mathrm{c}\end{array}$ \\
\hline $\begin{array}{l}\text { Paramètres } \\
\text { statistiques }\end{array}$ & & $\begin{array}{l}\text { ddl } \\
\text { F } \\
\text { P }\end{array}$ & $\begin{array}{l}5 \\
97,11 \\
<0,001\end{array}$ & $\begin{array}{l}5 \\
89,48 \\
<0,001\end{array}$ & $\begin{array}{l}\mathbf{5} \\
1619,13 \\
<\mathbf{0 , 0 0 1} \\
\end{array}$ \\
\hline
\end{tabular}

EAG : équivalent d'acide gallique, EQ : équivalent de quercétine, $\mathrm{EC}$ : équivalent de catéchine, SD : déviation standard, ddl : degré de liberté, F : statistique de Fisher, P : probabilité. Les valeurs avec les mêmes lettres dans chaque colonne ne sont pas significativement différentes $(\mathrm{p}<0,05)$. 


\section{Discussion}

Dans la présente étude, le potentiel antioxydant et les teneurs en polyphénols totaux, flavonoïdes et tanins condensés de deux espèces de la famille des Menispermaceae de Côte d'Ivoire ont été évalués. Les six extraits étudiés ont montré des activités antioxydantes. Les pourcentages d'inhibition du DPPH sont compris entre 33,84 et 69,21 \%. Selon Sahgal et al. (2009), même un faible pourcentage d'inhibition indique une certaine capacité de don de protons. Seule l'extrait acétate d'éthyle des feuilles de $A$. cordifolia $\left(\mathrm{CI}_{50}=\right.$ $\left.20 \mu \mathrm{g} . \mathrm{mL}^{-1}\right)$ a été plus actif que le trolox $\left(\mathrm{CI}_{50}=28 \mu \mathrm{g} \cdot \mathrm{mL}^{-1}\right)$. Les autres extraits, avec des $\mathrm{CI}_{50}$ largement supérieures à celle de la vitamine $\mathrm{C}$, ont été moins actifs. Toutefois, ces extraits pourraient servir d'inhibiteurs ou piégeurs de radicaux libres, agissant éventuellement comme des antioxydants primaires (Sahgal et al., 2009). Les extraits végétaux les plus actifs ont été les extraits acétate d'éthyle et éthanolique des feuilles de $A$. cordifolia suivis de l'extrait aqueux de $A$. scandens.

Tous les extraits ont une activité FRAP dose dépendante. Le pouvoir réducteur est proportionnel à l'augmentation des concentrations (Elmastas et al., 2006). L'activité observée indiquerait la présence de composés donneurs d'électrons dans les extraits étudiés, entraînant la réduction de $\mathrm{Fe}^{3+}$ en $\mathrm{Fe}^{2+}$. Selon Kumaran \& Karunakaran (2007), le pouvoir réducteur d'un composé peut servir d'indicateur significatif de son activité antioxydante potentielle. Tous les extraits étudiés possèdent donc une activité antioxydante. Les capacités de réduction du fer des extraits étudiés sont comprises entre 0,80 et 2,49 mgET.g ${ }^{-1} \mathrm{MS}$. Les capacités antioxydantes des extraits obtenus par la méthode de DPPH sont nettement supérieures à celles de la méthode de FRAP. Ceci pourrait être dû aux différences de sensibilité des réactifs utilisés dans chacune des méthodes (Ouedraogo et al., 2015). De plus, les activités antioxydantes des plantes varient d'un extrait à un autre. Selon Lafka et al. (2013), ces différences d'activité pourraient être attribuées aux divers composés extraits par les solvants de polarité différente. Dans l'ensemble, les résultats montrent que les extraits qui possèdent les meilleurs pouvoirs piégeurs vis-à-vis des radicaux libres $\mathrm{DPPH}$, montrent également les meilleures capacités réductrices du $\mathrm{Fe}^{3+}$. Cela indiquerait la présence dans les extraits analysés des molécules antioxydantes pouvant intervenir par deux types de mécanisme d'action. A notre connaissance, c'est la première fois que les propriétés antioxydantes de ces deux Menispermaceae sont rapportées. Néanmoins, l'activité antioxydante de plusieurs espèces de la famille des Menispermaceae a été déjà rapportée dans la littérature. C'est le cas de Tinospora cordifolia dont les extraits des feuilles avaient des teneurs en composés phénoliques abondantes et présentaient une activité antioxydante importante par le piégeage du radical DPPH, le pouvoir réducteur et la capacité de chélation des métaux (Praveen et al., 2012). Olorunnisola et al. (2011) ont 
montré que l'extrait méthanolique de l'écorce du tronc de Sphenocentrum jollyanum a une forte propriété antioxydante, qui pourrait être à l'origine de son action hépatoprotectrice dans les dommages hépatiques induits par le tétrachlorure de carbone. Plusieurs autres travaux ont indiqué le potentiel antioxydant des Menispermaceae (Amom et al., 2009; Ibahim et al., 2011). Les espèces de cette famille sont donc prometteuses dans la lutte contre le stress oxydant et ses conséquences dans l'organisme.

Les composés phénoliques constituent le groupe principal qui contribue à l'activité antioxydante des végétaux, fruits, céréales et d'autres produits à base de plantes (Tachakittirungrod et al., 2007). Ces derniers ont de multiples effets biologiques, y compris l'activité antioxydant (Rubió et al., 2013). Tous les extraits aqueux et organiques des feuilles de A. cordifolia, et des plantes entières de $A$. scandens ont montré des quantités significatives de composés phénoliques. L'activité antioxydante observée pourrait se justifier par la présence de ces composés.

Par ailleurs, des investigations phytochimiques ont mis en évidence la présence d'alcaloïdes dans ces plantes. L'alcaloïde, cycléanine déjà isolé de A. cordifolia est un excitant neuromusculaire à faible dose et un paralysant à forte dose (Debray et al., 1966). Quant à A. scandens, son étude chimique a permis d'isoler des alcalö̈des des feuilles et des graines (Philardeau, 1965). Selon $\mathrm{Ng}$ et al. (2000), les alcaloïdes sont doués de propriétés antioxydantes. Ainsi, l'activité antioxydante des extraits de A. cordifolia et A. scandens pourrait se justifier aussi par la présence de ces alcaloïdes non recherchés dans ce présent travail.

\section{Conclusion}

Ce travail avait pour objectif de déterminer le pouvoir antioxydant et la teneur en composés phénoliques des feuilles de $A$. cordifolia et des plantes entières de $A$. scandens deux espèces de la famille des Menispermaceae utilisées en médecine traditionnelle en Côte d'Ivoire. L'étude de l'activité antioxydante de ces deux espèces montre que les six extraits organiques et aqueux possèdent une activité antioxydante intéressante. Dans l'ensemble, ces activités restent nettement inférieures à celles de l'acide ascorbique et du trolox. Elles pourraient être améliorées et probablement se rapprocher de celles des standards, avec la purification des molécules actives. Des recherches complémentaires sont nécessaires pour identifier, isoler et purifier les constituants actifs.

\section{Remerciements}

Nous remercions sincèrement le Centre Suisse de Recherches Scientifiques en Côte d'Ivoire (CSRS) pour l'assistance technique. 


\section{References:}

1. Afsar, T., Razak, S., Shabbir, M., \& Khan, M. R. (2018). Antioxidant activity of polyphenolic compounds isolated from ethyl-acetate fraction of Acacia hydaspica R. Parker. Chemistry Central Journal, 12(1), 5 .

2. Amom, Z., Bahari, H., Isemaail, S., Ismail, N. A., Shah, Z. M., \& Arsyad, M. S. (2009). Nutritional composition, antioxidant ability and flavonoid content of Tinospora crispa stem. Advances in Natural and Applied Sciences, 3(1), 88-95.

3. Aseervatham, G. S. B., Sivasudha, T., Jeyadevi, R., \& Ananth, D. A. (2013). Environmental factors and unhealthy lifestyle influence oxidative stress in humans - an overview. Environmental Science and Pollution Research, 20(7), 4356-4369.

4. Ba, K., Tine, E., Destain, J., Cissé, N., \& Thonart, P. (2010). Étude comparative des composés phénoliques, du pouvoir antioxydant de différentes variétés de sorgho sénégalais et des enzymes amylolytiques de leur malt. Biotechnologie, Agronomie, Société et Environnement, 14(1), 131-139.

5. Bahorun, T., Gressier, B., Trotin, F., Brunet, C., Dine, T., Luyckx, M., \& Pinkas, M. (1996). Oxygen species scavenging activity of phenolic extracts from hawthorn fresh plant organs and pharmaceutical preparations. Arzneimittel-forschung, 46(11), 1086-1089.

6. Bokhari, J., Khan, M. R., Shabbir, M., Rashid, U., Jan, S., \& Zai, J. A. (2013). Evaluation of diverse antioxidant activities of Galium aparine. Spectrochimica Acta Part A: Molecular and Biomolecular Spectroscopy, 102, 24-29.

7. Bouquet, A. \& Debray, M. (1974). Plantes médicinales de la Côte d'Ivoire. Travaux et Documents No 32. ORSTOM, Paris (France), 231 pp.

8. Debray, M., Plat, M. \& Le Men, J. (1966). Alcaloïdes des Menispermacees africaines Epinetrum cordifolium et Epinetrum mangenotii: isolement de la cycleanine, de la nor-cycleanine et de l'isochondrodendrine. Annales Pharmaceutiques Françaises, 24(7-8), 551-558.

9. Elmastas, M., Turkekul, I., Ozturk, L., Gulcin, I., Isildak, O., \& AboulEnein, H. Y. (2006). Antioxidant activity of two wild edible mushrooms (Morchella vulgaris and Morchella esculanta) from North Turkey. Combinatorial Chemistry \& Highthroughput Screening, 9(6), 443-448.

10. Favier, A. (2003). Le stress oxydant, intérêt conceptuel et expérimental dans les compréhensions des mécanismes des maladies et potentiel thérapeutiques. L'actualité chimique, 270, 108-115. 
11. Ibahim, M. J., Wan-Nor, I. W. M. Z., Narimah, A. H. H., SAR, S. N. S., \& Froemming, G. A. (2011). Anti-proliperative and antioxidant effects of Tinospora crispa (Batawali). Biomedical research, 22(1).

12. Kaur, P., Singh, B., Kumar, S., \& Kaur, S. (2008). In vitro evaluation of free radical scavenging activity of Rubia cordifolia L. Journal of Chinese clinical medicine, 3(5), 278-284.

13. Kicel, A., Michel, P., Owczarek, A., Marchelak, A., Żyżelewicz, D., Budryn, G., \& Olszewska, M. A. (2016). Phenolic profile and antioxidant potential of leaves from selected Cotoneaster Medik. Species. Molecules, 21(6), 688.

14. Konan, Y., Witabouna, K. M., Bassirou, B., \& Kagoyire, K. (2014). Antioxidant activity and total phenolic content of nine plants from Côte d'Ivoire (West Africa). Journal of Applied Pharmaceutical Science, 4(8), 36.

15. Kosanić M. \& Ranković B. (2011). Lichens as possible sources of antioxidants. Pakistan Journal of Pharmaceutical Sciences, 24(2), 165-170.

16. Kumaran, A., \& Karunakaran, R. J. (2007). In vitro antioxidant activities of methanol extracts of five Phyllanthus species from India. LWT-Food Science and Technology, 40(2), 344-352.

17. Lafka, T. I., Lazou, A. E., Sinanoglou, V. J., \& Lazos, E. S. (2013). Phenolic extracts from wild olive leaves and their potential as edible oils antioxidants. Foods, 2(1), 18-31.

18. Liu, Z., Mo, K., Fei, S., Zu, Y., \& Yang, L. (2017). Efficient approach for the extraction of proanthocyanidins from Cinnamomum longepaniculatum leaves using ultrasonic irradiation and an evaluation of their inhibition activity on digestive enzymes and antioxidant activity in vitro. Journal of separation science, 40(15), 3100-3113.

19. Liu, Z., \& Yang, L. (2018). Antisolvent precipitation for the preparation of high polymeric procyanidin nanoparticles under ultrasonication and evaluation of their antioxidant activity in vitro. Ultrasonics sonochemistry, 43, 208-218.

20. Magder, S. (2006). Reactive oxygen species: toxic molecules or spark of life? Critical Care, 10(1), 208.

21. Meena, J., \& Santhy, K. S. (2015). Evaluation of the antioxidant potential of methanol extract of Cyclea peltata in DAL Model. Pharm Innovat $\mathrm{J}, 4,71-75$.

22. Ng, T. B., Liu, F., \& Wang, Z. T. (2000). Antioxidative activity of natural products from plants. Life sciences, 66(8), 709-723.

23. Olorunnisola, O. S., Akintola, A. O., \& Afolayan, A. J. (2011). Hepatoprotective and antioxidant effect of Sphenocentrum jollyanum (Menispermaceae) stem bark extract against $\mathrm{CCl}_{4}$-induced oxidative 
stress in rats. African Journal of Pharmacy and Pharmacology, 5(9), 1241-1246.

24. Ouedraogo, R. A., Koala, M., Dabire, C., Hema, A., Bazie, V. B. E. J. T., Outtara, L. P., \& Nebie, R. H. (2015). Teneur en phénols totaux et activité antioxydante des extraits des trois principales variétés d'oignons (Allium cepa L.) cultivées dans la région du Centre-Nord du Burkina Faso. International Journal of Biological and Chemical Sciences, 9(1), 281-291.

25. Oyaizu, M. (1986). Studies on products of browning reaction. The Japanese journal of nutrition and dietetics, 44(6), 307-315.

26. Praveen, N., Thiruvengadam, M., Kim, H. J., Kumar, J. P., \& Chung, I. M. (2012). Antioxidant activity of Tinospora cordifolia leaf extracts through non-enzymatic method. Journal of Medicinal Plants Research, 6(33), 4790-479.

27. Philardeau, Y., \& Debray, M. M. (1965). Note Preliminaire Sur les Alcaloïdes de Sphenocentrum jollyanum. Rapport Roneeo ORSTOM. Plantes Médicinales De La Côte D'Ivoire, Bouquet, A. and M. Debray (Eds.). ORSTOM, Paris (France), 115.

28. Rubió, L., Motilva, M. J., \& Romero, M. P. (2013). Recent advances in biologically active compounds in herbs and spices: a review of the most effective antioxidant and anti-inflammatory active principles. Critical reviews in food science and nutrition, 53(9), 943-953.

29. Sahgal, G., Ramanathan, S., Sasidharan, S., Mordi, M. N., Ismail, S., \& Mansor, S. M. (2009). In vitro antioxidant and xanthine oxidase inhibitory activities of methanolic Swietenia mahagoni seed extracts. Molecules, 14(11), 4476-4485.

30. Singleton, V. L., \& Rossi, J. A. (1965). Colorimetry of total phenolics with phosphomolybdic-phosphotungstic acid reagents. American journal of Enology and Viticulture, 16(3), 144-158.

31. Sohal, R. S., Mockett, R. J., \& Orr, W. C. (2002). Mechanisms of aging: an appraisal of the oxidative stress hypothesis1, 2. Free Radical Biology and Medicine, 33(5), 575-586.

32. Tachakittirungrod, S., Ikegami, F., \& Okonogi, S. (2007). Antioxidant active principles isolated from Psidium guajava grown in Thailand. Scientia pharmaceutica, 75(4), 179.

33. Wang, Y. Z., Fu, S. G., Wang, S. Y., Yang, D. J., Wu, Y. H. S., \& Chen, Y. C. (2018). Effects of a natural antioxidant, polyphenol-rich rosemary (Rosmarinus officinalis L.) extract, on lipid stability of plantderived omega-3 fatty-acid rich oil. LWT-Food Science and Technology, 89, 210-216. 
34. Wangcharoen, W., \& Morasuk, W. (2007). Antioxidant capacity and phenolic content of some Thai culinary plants. Maejo International Journal of Science and Technology, 1(2), 100-106.

35. Westlake, W. J. (1971). A one-sided version of the Tukey-Duckworth test. Technometrics, 13(4), 901-903.

36. ZulkEfli, H. N., Mohamad, J., \& Abidin, N. Z. (2013). Antioxidant activity of methanol extract of Tinospora crispa and Tabernaemontana corymbosa. Sains Malaysiana, 42(6), 697-706. 